\title{
Descripción Morfológica e Histológica del Tejido Hepático de Gambusia holbrooki (Pisce: Poeciliidae) que Habita en la Provincia de Arica-Chile
}

\author{
Morphological and Histological Description of the Liver Tissue of Gambusia \\ holbrooki (Pisce: Poeciliidae) Inhabiting the Province of Arica-Chile
}

José López-Cepeda ${ }^{1}$; Nicolás Garrido ${ }^{1,3}$; Hipólito Núñez ${ }^{1}$ Arnaldo Vilaxa-Olcay ${ }^{1,2}$

LÓPEZ-CEPEDA, J.; GARRIDO, N.; NÚÑEZ, H. \& VILAXA-OLCAY, A. Descripción morfológica e histológica del tejido hepático

de Gambusia holbrooki (Pisce: Poeciliidae) que habita en la provincia de Arica - Chile. Int. J. Morphol., 38(3):645-649, 2020.

RESUMEN: Gambusia holbrooki es el pez de agua dulce con mayor distribución en Chile y el mundo, pero los estudios que abordan la morfología e histología hepática del pez son escasos. El hígado es utilizado para evidenciar efectos subletales de contaminantes ambientales y es preciso contar con una descripción histomorfológica del hígado para futuros estudios comparativos. El presente estudio tiene como objetivo describir patrones histomorfológicos del hígado de Gambusia holbrooki, para ello se colectaron 97 individuos adultos de los sistemas límnicos de los valles de Lluta y Azapa (Extremo norte de Chile), para observar patrones morfológicos comunes en ambas poblaciones, se utilizó técnicas histológicas de rutina e histoquímica. Las evidencias demostraron que el hígado contiene tejido pancreático y su arquitectura tisular es trabecular con mayor presencia de capilares sinusoides.

PALABRAS CLAVE: Morfología; Histología; Hepatopáncreas; Pez mosquito.

\section{INTRODUCCIÓN}

En la mayoría de los teleósteos el hígado es un órgano situado en la porción anterior de la cavidad abdominal y cumple funciones similares a los mamíferos; incluyen la asimilación de nutrientes, producción de bilis, desintoxicación, mantenimiento de la homeostasis metabólica del cuerpo que incluye procesamiento de carbohidratos, proteínas, lípidos y vitaminas (Arellano \& Sarasquete, 2006). El hígado también desempeña un papel clave en la síntesis de proteínas plasmáticas, como la albúmina y fibrinógeno (Genten et al., 2009), siendo un órgano vulnerable al estrés crónico (Zacarias et al., 2009) o la contaminación ambiental (Abdel-Moneim et al., 2012).

La arquitectura hepática varía entre las especies, y en una misma especie puede variar a causa del ciclo reproductivo en la formación de la vitelogenina, hormona que propicia la síntesis del líquido nutricional (vitelo) para los embriones durante el periodo reproductivo de las hembras y también de la cantidad de depósito de reserva como las grasas y glucógeno; que dependerá de la calidad y cantidad de alimento ingerido y metabolizado (Arellano \& Sarasquete).

En los peces la histología del hígado se diferencian de los mamíferos porque hay menos tendencia a la disposición de lobulillos hepáticos y las tríadas portales típicas son raramente vistas; Los capilares sinusoides están revestidos con células endoteliales con núcleos alargados sobresaliendo el lumen sinusoidal (Genten et al.). La estructura acinar del páncreas exocrino de los teleósteos puede estar asociada al hígado y es bastante sencilla; las unidades terminales secretoras (ácinos) están constituidas por células secretoras de enzimas y su estructura corresponde a cimógenos (Arellano \& Sarasquete).

Considerando que la morfología hepática puede variar por procesos biológicos naturales o en respuesta a la contaminación, el presente estudio identifica patrones histomorfológicos hepáticos de dos poblaciones que residen en dos hábitat aislados como son el valle de Lluta y Azapa, región árida del norte de Chile (López-Cepeda et al., 2014).

\footnotetext{
${ }^{1}$ Departamento de Biología, Facultad de Ciencias, Universidad de Tarapacá, Avda. General Velásquez 1775, Arica, Chile.

${ }^{2}$ Centro de Estudios Ciencias Marinos y Limnológicos, Facultad de Ciencias, Universidad de Tarapacá, Avda. General Velásquez 1775, Arica, Chile.

${ }^{3}$ Laboratorio de Genética y Patogénesis Bacteriana, Facultad de Ciencias de la Vida, Universidad Andrés Bello, República 239, Santiago Chile.
} 


\section{MATERIAL Y MÉTODO}

Para observar patrones hepáticos comunes se colectaron de una sola vez 97 individuos adultos ( 57 hembras y 40 machos) de estanques acumuladores de agua ubicados en los valles de Lluta y Azapa. Las aguas que se acumulan en el valle de Lluta tiene un alta salinidad, con una conductividad eléctrica sobre $2 \mathrm{ds} / \mathrm{m}$, en cambio la conductividad eléctrica del agua que se acumula en el valle de Azapa, que puede ser de la cuenca del río San José o la trasvasada desde la cuenca del río Lauca, en cualquier caso, es menor a $1 \mathrm{ds} / \mathrm{m}$ (Torres \& Acevedo, 2008).

Los peces capturados fueron llevados vivos al laboratorio de Microbiología e Histología de la Universidad de Tarapacá, se anestesiaron con benzocaína $(0,5 \mathrm{~g} / \mathrm{L})$ para ser sacrificados por punción medular. Fue disecado el hígado de cada uno de en todos los peces y procesados para su observación en microscopía óptica, el órgano completo fue fijado en Buin - Hollande y deshidratado en alcoholes a partir de $70 \%$, se aclararon en xilol y se incluyeron en parafina.
Las muestras fueron cortadas a 7 y 8 micras de grosor. Los cortes se hidrataron y tiñeron con Hematoxilina - Eosina, Picrofucsina de Van Gieson considerada como la coloración más selectiva de las fibras colágenas y P.A.S (Periodic Acid Schiff), para detectar carbohidratos (Torres, 2002).

El estudio morfológico y las fotografías se realizaron con un microscopio óptico marca Olympus modelo CX31. Para evidenciar patrones hepáticos se utilizó el siguiente criterio: 1) integridad del hepatocito, se debe observar claramente núcleo y límite celular, 2) vasos sanguíneos continuos y en su interior eritrocitos, 3 ) epitelios claramente definidos y por último 4) se descartó toda muestra que presentara alguna anomalía o posible patología.

\section{RESULTADOS}

El hígado en Gambusia holbrooki fue localizado en la región media y anterior de la cavidad abdominal, por encima y al costado izquierdo del estómago e intestino, no se logra
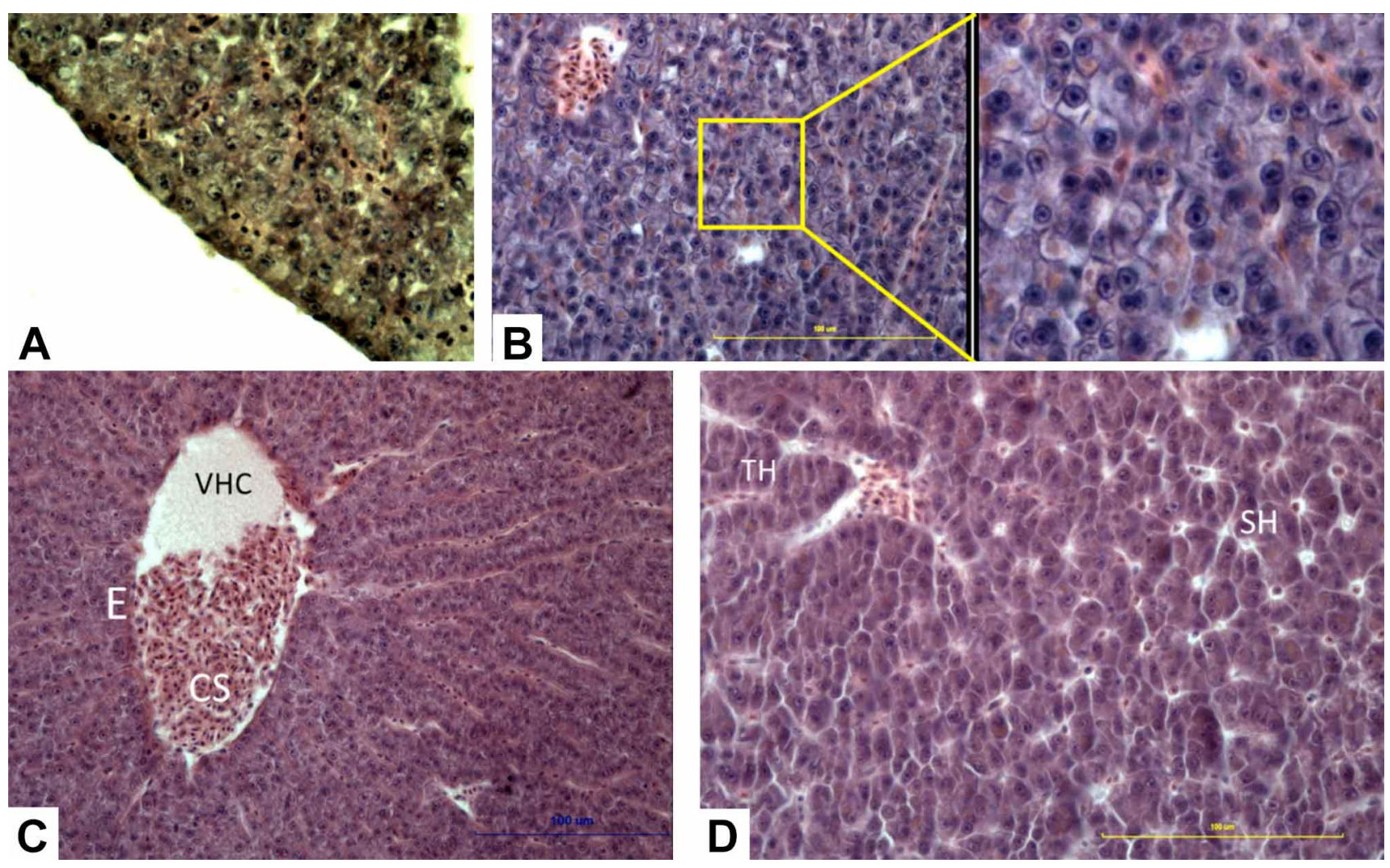

Fig. 1) Corresponde a una serie de cortes histológicos de hígados, obtenidas a 400x. En la imagen A se observa la capsula que envuelve al tejido hepático (Van Gieson). Imagen B se observa el parénquima hepático con una ampliación a los hepatocitos (Hematoxilina Eosina). Imagen C Corresponde a una gran vena hepática central (VHC) con células sanguíneas (CS) rodeadas de un endotelio (E); mientras que, en la imagen D se puede apreciar las trabéculas hepáticas constituidas por los hepatocitos (TH), entre ellas se observa con claridad los sinusoides hepáticos (SH). En general no hay una delimitación clara del lobulillo hepáticos por tejido conjuntivo (Hematoxilina Eosina). 
observar bajo lupa la división anatómica de lóbulos hepáticos, más bien tiene un aspecto de disco y textura lisa, el tamaño del hígado corresponde a 1/4 del tamaño corporal del pez.

Se reconoce una fina cápsula que envuelve al hígado, compuesta por epitelio simple plano más tejido conjuntivo basal carente de fibras colágenas, hacia el interior se observa el parénquima hepático (Fig. 1 A). Los hepatocitos del parénquima mantienen una forma poliédrica, debido a que están agrupados en cordones, se observa en ellos un citoplasma; cada hepatocito presenta un núcleo central y redondo, además de un vistoso nucléolo homogéneo (Fig. 1 B). El tejido conjuntivo es muy escaso, en algunos casos se observa presencia de fibras colágenas seguramente asociado a la capa media y adventicia de los vasos sanguíneos. La disposición de los hepatocitos es en tabiques formando cordones agregados; los espacios que dejan las trabéculas hepáticas son ocupados por capilares sinusoides en el cual circulan eritrocitos nucleados (Fig. $1 \mathrm{C}$ ).

Poca tendencia a la disposición de lobulillo clásico, con forma hexagonal, debido a que son escasas las venas centrolobulillares, sin embargo, se observan agrupaciones de hepatocitos rodeando capilares sinusoides, dando la apariencia de pequeños lobulillos hepáticos (Fig. 1 D), sin la asociación de las tríadas portales típicas. Este hecho imposibilita observar unidades morfofuncionales como los lobulillos porta o ácinos hepáticos. Se observan arteriolas y vénulas rodeadas por un tejido conjuntivo laxo en distintas secciones del hígado, sin un patrón definido. No se observa conducto biliar.

Integrado al tejido hepático se reconoce el páncreas como un epitelio simple cúbico alto, que forma un conducto que va desde el costado del hígado hasta el interior del órgano, el cual corresponde a un tubo altamente irrigado, existe un tejido conjuntivo laxo entre el epitelio simple cúbico y el parénquima hepático, en su interior circulan eritrocitos y un líquido de naturaleza básica frente a la tinción de eosina. Cada célula cúbica presenta un núcleo basal y homogéneo con un nucléolo heterocrómico, el citoplasma de la célula cúbica es más teñida hacia la base y menos teñida hacia la luz del tubo, esto se debe que presenta cimógeno en forma de pequeños gránulos, que tiñen rosado en Hematoxilina - Eosina y Picrofucsina de Van Gieson y transparente frente a la reacción de P.A.S. (Fig. 2).
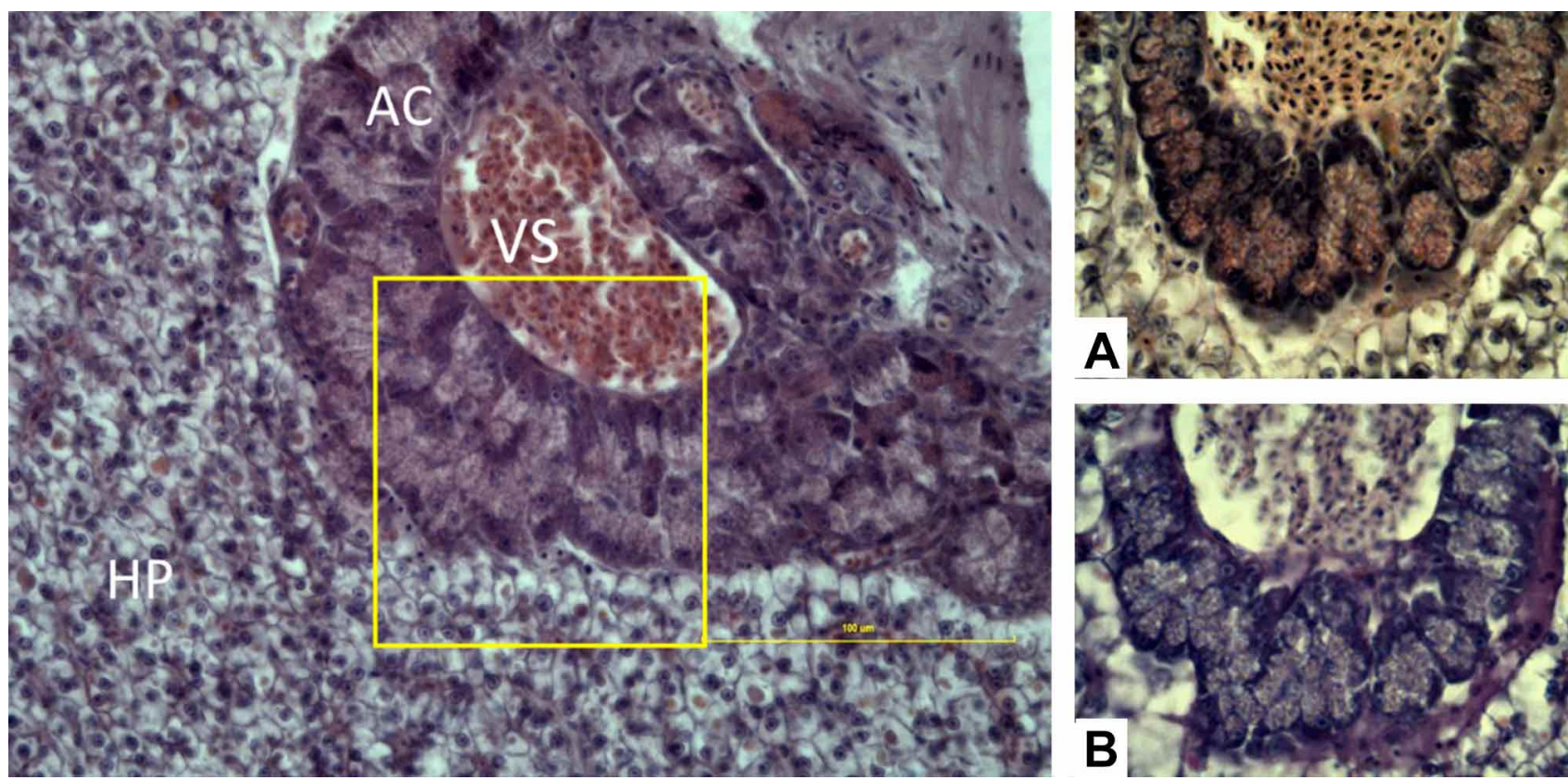

Fig. 2) Corresponde al Hepatopáncreas, se puede observar al tejido hepático (HP) y el tejido pancreático distribuido en acinos o masas celulares pancreáticas (AC). Al centro un gran vaso sanguíneo (VS) con células sanguíneas rodeadas de endotelio, entre los acinos pancreáticos se puede observar vasos sanguíneos de menor calibre (vénulas, arteriolas). (Hematoxilina Eosina), imagen A y B ampliación del tejido pancreático teñidos con Van Gieson y PAS, respectivamente.

\section{DISCUSIÓN}

El hígado de Gambusia holbrooki está ordenada por medio de trabéculas hepáticas intercaladas con sinusoides, coincidentes con otras especies Gnathonemus petersii, Rutilus rutilus y Syprinus carpio (Genten et al.), mientras 
que la morfología poliédrica de los hepatocitos con núcleo central y nucléolo vistoso es común a muchas especies de teleósteos, por ejemplo Oreochromis niloticus (Torres et al., 2010), Paralabrax maculatofasciatus (Zacarias et al.), Schilbe mytus, Labeo niloticus (Naguid et al., 2009) у Scatophagus argus (Morovvati et al., 2012). Estas coincidencias morfológicas en especies tan distintas filogenéticamente, permite sostener que la morfología hepática se perpetua con la función, posibilitando diferenciar estructuras histológicas para estudios biométricos utilizando unidades como el tamaño y número de los hepatocitos o variación en la irrigación sanguínea del hígado.

El análisis comparativo de las dos poblaciones en estudio permite obtener el siguiente patrón morfohistológico, el hígado está constituido por un estroma y parénquima. El estroma corresponde al tejido de sostén como la cápsula que recubre, protege e individualiza al órgano; en peces corresponde a un tejido conjuntivo fibroso (Genten et al.), mientras que para G. holbrooki se observó en todos los casos un epitelio simple plano. Desde la cápsula parten trabéculas hepáticas hacia el interior que es sostenido por un escaso tejido conjuntivo. El parénquima está representado por dos componentes anatómicos: hepático y pancreático.

El componente hepático está dado por los hepatocitos, que forman cordones con orientación radial, que se dirigen hacia la vena centrolobulillar y un sistema vascular que mantiene el flujo sanguíneo a través de los capilares sinusoides que provienen de la vena portal y arteria hepática drenando el contenido a la vena centrolobulillar, lo más probable que la unidad morfológica del hígado de G. holbrooki sean pequeños ácinos hepáticos rodeando un capilar sinusoide, ya que no presenta la unidad hexagonal de lobulillo hepático, a diferencia con lo notificado por Torres et al. para Tilapia (Oreochromis niloticus). Tampoco se observan triadas portales, por lo tanto el lobulillo hepático sería un argumento débil a la hora de hacer estudios biométricos en el pez mosquito.

El componente pancreático del parénquima hepático de $G$. holbrooki coincide con lo informado por Petcoff et al. (2006) para Oligosarcus jenynsii, el cual asocia el páncreas exocrino como un arreglo acinar de células piramidales que rodean a la vena portal, esto se evidencia al observar eritrocitos en el lumen pancreático. Cada célula serosa contiene en su citoplasma gránulos con abundantes proenzimas digestivas (cimógenos) que se activan en la luz intestinal. Estas sustancias son secretadas por exocitosis en su membrana apical hacia un vaso sanguíneo al centro de la luz acinar; en ella se visualiza el endotelio vascular (Torres et al.).
Este análisis morfológico del hígado de dos poblaciones de peces que habitan en ambientes muy distintos permitió establecer patrones histológicos comunes que contribuyen al conocimiento de la biología del pez, pero además permite precisar elementos estructurales primarios que demostraran un eventual efecto al estrés ambiental, tanto natural como antrópico.

LÓPEZ-CEPEDA, J.; GARRIDO, N.; NÚÑEZ, H. \& VILAXA-OLCAY, A. Morphological and histological description of the liver tissue of Gambusia holbrooki (Pisce: Poeciliidae) inhabiting the province of Arica - Chile. Int. J. Morphol., 38(3):645-649, 2020.

SUMMARY: Gambusia holbrooki is the freshwater fish with the greatest distribution in Chile and the world. However, studies dealing with morphology and liver histology of fish are scarce. The liver commonly shows the sublethal effects of environmental pollutants and there should be a histomorphological description of the liver for further comparative studies. The present study aims to describe histomorphological patterns of the liver of Gambusia holbrooki. A total of 97 adult individual specimens were collected from the ecosystems in the valleys of Lluta and Azapa (Region of Arica and Parinacota), to observe patterns morphologically common in both populations. Routine histological and histochemical techniques were used for analysis. The evidence showed that the liver contains pancreatic tissue, and that tissue architecture is trabecular with greater presence of capillary sinusoids.

KEY WORDS: Morphology; Histology; Hepatopancreas; Mosquitofish.

\section{REFERENCIAS BIBLIOGRÁFICAS}

Abdel-Moneim, A. M.; Al-Kahtani, M. A. \& Elmenshawy, O. M. Histopathological biomarkers in gills and liver of Oreochromis niloticus from polluted wetland environments, Saudi Arabia. Chemosphere, 88(8):1028-35, 2012.

Arellano, J. \& Sarasquete, C. Atlas Histológico del Lenguado Senegalés Solea Senegalensis (Kauo, 1858). Madrid, Sociedad Anónima de Fotocomposición, 2006.

Genten, F.; Terwinghe, E. \& Danguy, A. Atlas of Fiish Histology. New Hampshire, Science Publishers, 2009.

López-Cepeda, J.; Pacheco-Cartagena, P. \& Vilaxa-Olcay, A. Diferencias morfométricas de Gambusia holbrooki (Pisce: Poeciliidae) que habitan en los estanques de regadío de los valles de Lluta y Azapa, Chile. Idesia (Arica), 32(3):59-64, 2014.

Morovvati, H.; Nikpour, Y.; Zolgharneine, H.; Ronagh, M.; Abdi, R. \& Roshan, A. Histological changes in the liver of reared spotted scat (Sactophagus argatus L.) after exposure to mercury. Comp. Clin. Pathol., 21:745-53, 2012.

Naguid, S. A.; Rizkalla, W. \& Abd El Ghafar, F. A. Comparative histological and ultraestructural studies on the liver and páncreas of Schilbe mytus and Labeo nicloticus. Egypt. J. Aquat. Biol. Fish., 3(1):107-27, 2009. 
Petcoff, G. M.; Díaz, A. O.; Escalante, A. H. \& Goldemberg, A. L. Histology of the liver of Oligosarcus jenynsii (Ostariophysi, Characidae) from Los Padres Lake, Argentina. Iheringia Ser. Zool., 96(2):205-8, 2006.

Torres, F. Manual de Técnicas en Histología y Anatomía Patológica. Barcelona, Editorial Ariel, 2002.

Torres, H. A. \& Acevedo, H. E. El problema de salinidad en los recursos suelo y agua que afectan el riego y cultivos en los valles de Lluta y Azapa en el norte de Chile. Idesia (Arica), 26(3):31-44, 2008.

Torres, R. G. A.; González, P. S. \& Peña, S. E. Anatomical, Histological and ultraestructural description of the gills and liver of the tilapia (Oreochromis niloticus). Int. J. Morphol., 28(3):703-12, 2010.

Zacarias, M.; Cadena, M. \& Rivas, P. Structural modifications in the stomach and liver of Paralabrax maculato fasciatus (Steindacher, 1868) under chronic stress conditions. Int. J. Morphol., 27(2):425-33, 2009.

\author{
Direccion para correpondencia: \\ Dr. Arnaldo Vilaxa \\ Departamento de Biología \\ Facultad de Ciencias \\ Universidad de Tarapacá \\ Avda. General Velásquez 1775 \\ Arica \\ CHILE
}

Email: avilaxa@uta.cl

Recibido : 11-08-2019

Aceptado: 03-01-2020 